\title{
SEMI-EMBEDDINGS OF BANACH SPACES WHICH ARE HEREDITARILY $c_{0}$
}

\author{
by L. DREWNOWSKI
}

(Received 5th November 1981)

To Professor W. Orlicz on his 80th birthday

Following Lotz, Peck and Porta [9], a continuous linear operator from one Banach space into another is called a semi-embedding if it is one-to-one and maps the closed unit ball of the domain onto a closed (hence complete) set. (Below we shall allow the codomain to be an $F$-space, i.e., a complete metrisable topological vector space.) One of the main results established in [9] is that if $X$ is a compact scattered space, then every semi-embedding of $C(X)$ into another Banach space is an isomorphism ([9], Main Theorem, $(a) \Rightarrow(b))$.

In this paper we extend this result, as well as ([9], Corollary 12), to those Banach spaces $E$ which are hereditarily $c_{0}$, i.e., such that every closed infinite-dimensional subspace of $E$ contains a further subspace that is isomorphic to $c_{0}$. Theorem 1 proved below shows, in particular, that every semi-embedding of such a space $E$ into any $F$ space is an isomorphism. Note that the class of hereditarily $c_{0}$ Banach spaces includes all spaces isomorphic to the $C(X)$ spaces for $X$ compact scattered ([10], Main Theorem, (5); cf. also [9], Main Theorem 11, (d)), in particular all $c_{0}(\Gamma)$ spaces, and the separable Banach space constructed by Hagler [5]. Trivially, if $E$ is hereditarily $c_{0}$, then so is every closed subspace of $E$. It should be pointed out, however, that Hagler's space is not isomorphic to a subspace of $C(X)$ for $X$ compact scattered; this follows from ([5], Proposition 13) and ([10], Main Theorem, (4)).

Our second result, Theorem 2 , is, in a sense, a converse to the above mentioned special case of Theorem 1. It reveals, quite unexpectedly, that the existence of a nonhereditarily $c_{0}$ Banach space $E$ such that, for $E$ equipped with any of its equivalent norms, every semi-embedding of $E$ is an isomorphism, would imply a negative solution to the following well-known and long-standing problem of Bessaga and Pełczyński ([7], Problem 1.d.5): Does every infinite-dimensional Banach space contain an unconditional basic sequence?

Theorem 1. Let $E=(E,\|\cdot\|)$ be a Banach space and $F$ its closed subspace which is hereditarily $c_{0}$. Suppose that

$(+)$ for every closed infinite-dimensional subspace $G$ of $F$ there is a complemented subspace $H$ of $E$ such that $\operatorname{dim}(G \cap H)=\infty$ and $H$ has no subspace isomorphic to $l_{\infty}$.

Then, $T$ is a semi-embedding of $E$ into an $F$-space, implies that $T \mid F$ is an isomorphism. Equivalently: If $|\cdot|$ is a weaker F-norm (see [6]) on (E, $\|\cdot\|)$ such that the closed unit ball $B$ of $(E,\|\cdot\|)$ is complete under $|\cdot|$, then $|\cdot|$ and $\|\cdot\|$ are equivalent on $F$. 
Proof. Suppose an $F$-norm $|\cdot|$ satisfies the above assumptions and is strictly weaker than $\|\cdot\|$ on $F$. We first show that $F$ has a norm-closed infinite-dimensional subspace $G$ such that $|\cdot|$ is strictly weaker than $\|\cdot\|$ on every infinite-dimensional subspace of $G$.

Since $B$ is $|\cdot|$-complete, and a fortiori $|\cdot|$-closed, the norm topology of $F$ is polar with respect to the (strictly weaker) $|\cdot|$-topology of $F$. Hence, by a result of Kalton ([6], Theorem 3.2 or [2], Theorem 2.4), we may find a normalised basic sequence $\left(u_{n}\right)$ in $(F,\|\cdot\|)$ such that $\sum_{n=1}^{\infty}\left|u_{n}\right|<\infty$. Let $G$ be the norm-closed linear span of $\left(u_{n}\right)$, and let $\left(f_{n}\right) \subset(G,\|\cdot\|)^{*}$ be the associated sequence bi-orthogonal to $\left(u_{n}\right)$. Then sup $\left\|f_{n}\right\|<\infty$, from which it follows easily that

$$
\left|x-\sum_{i=1}^{n} f_{i}(x) u_{i}\right| \rightarrow 0 \quad \text { as } \quad n \rightarrow \infty,
$$

uniformly for $x \in B \cap G$. Hence the identity map from $(G,\|\cdot\|)$ into $(E,|\cdot|)$ is compact. It follows that $G$ is as required.

Now, by applying the hypotheses of the theorem and passing to a suitable subspace of $G$, we may assume that $G$ is isomorphic to $c_{0}$ and that $G$ is contained in a complemented subspace $H$ of $E$ such that $H \not l_{\infty}$. Moreover, using a suitable isomorphism, we may identify $G$ with $c_{0}$ so as to have $\|\cdot\| \leqq\|\cdot\|_{\infty}$ on $G$, where $\|\cdot\|_{\infty}$ is the usual sup-norm of $c_{0}$.

Let $\left(e_{n}\right)$ be the standard basis of $c_{0}=G$. Since, for each $n,|\cdot|$ is strictly weaker than $\|\cdot\|$ (or $\left.\|\cdot\|_{\infty}\right)$ on $\operatorname{lin}\left\{e_{i}: i \geqq n\right\}$, there is a block sequence $\left(x_{n}\right)$ of $\left(e_{n}\right)$ such that $\left\|x_{n}\right\|_{\infty}=1$ for all $n$ and

$$
\sum_{n=1}^{\infty}\left|x_{n}\right|<\infty
$$

Let $\left(t_{n}\right) \in l_{\infty}$ and $\left\|\left(t_{n}\right)\right\|_{\infty} \leqq 1$. Since the "supports" of the $x_{n}$ 's are pairwise disjoint, we have

$$
\sum_{i=1}^{n} t_{i} x_{i} \in B \text { for all } n
$$

furthermore,

$$
\left|\sum_{i=m}^{n} t_{i} x_{i}\right| \leqq \sum_{i=m}^{n}\left|x_{i}\right| \rightarrow 0 \quad \text { as } m, n \rightarrow \infty
$$

Since $B$ is $|\cdot|$-complete, the series $\sum_{n=1}^{\infty} t_{n} x_{n}$ is (subseries) convergent in $(E,|\cdot|)$ to a point in $B$.

Let $P$ be a continuous linear projection from $E$ onto $H$. We will indicate two ways of finishing the proof.

(I) From the above it follows that we may define a continuous linear operator 
$R: l_{\infty} \rightarrow(E,\|\cdot\|)$ by

$$
R\left(t_{n}\right)=\sum_{n=1}^{\infty} t_{n} x_{n} \quad(|\cdot| \text {-convergence })
$$

Let $S=P R: l_{\infty} \rightarrow(H,\|\cdot\|)$. Since the basic sequence $\left(x_{n}\right)$ is equivalent to the basis $\left(e_{n}\right)$ of $c_{0}$ ([7], Proposition 2.a.1), $S\left|c_{0}=R\right| c_{0}$ is an isomorphism. By a result of Rosenthal ([11], Proposition 1.2; cf. also [3] and [4]), there is an infinite subset $M$ of $\mathbb{N}=\{1,2, \ldots\}$ such that the restriction of $S$ to the subspace $l_{\infty}(M)=\left\{\left(t_{n}\right) \in l_{\infty}: t_{n}=0\right.$ for $\left.n \notin M\right\} \approx l_{\infty}$ is an isomorphism. This is clearly impossible because $H \not l_{\infty}$.

(II) From the above we see that the series $\sum_{n=1}^{\infty} x_{n}$ is subseries convergent in $(E,|\cdot|)$, and that the sum of each of its subseries is in $B$. We may therefore define a bounded finitely additive vector measure $\mu: 2^{\mathrm{N}} \rightarrow(H,\|\cdot\|)$ by

$$
\mu(A)=P\left(\sum_{n \in A} x_{n}\right) \quad(|\cdot| \text {-convergence }) .
$$

Since $H \not l_{\infty}$, a theorem of Diestel and Faires ([1], Theorem I.4.2) implies $\mu$ is exhaustive, i.e., $\left\|\mu\left(A_{n}\right)\right\| \rightarrow 0$ for every infinite sequence $\left(A_{n}\right)$ of pairwise disjoint subsets of $\mathbb{N}$. In particular as $\mu(\{n\})=x_{n}$, we have $\left\|x_{n}\right\| \rightarrow 0$, contradicting the fact that $\left(x_{n}\right)$ was chosen normalised in the norm $\|\cdot\|_{\infty}$ equivalent to $\|\cdot\|$ on $G$.

Remarks. (1) Since the requirements imposed in Theorem 1 on the Banach space $E$ and its subspace $F$ are isomorphism invariants, it is clear that the assertion remains valid for $E$ equipped with any of its equivalent norms.

(2) The condition $(+)$ is satisfied in particular when

(a) $E \not l_{\infty}$, or

(b) $F$ is complemented in $E$, or

(c) every subspace of $F$ isomorphic to $c_{0}$ contains a further subspace which is isomorphic to $c_{0}$ and complemented in $E$.

The simplest case when $(a)$ or $(b)$ holds is $E=F$.

Theorem 2. A Banach space $E$ is hereditarily $c_{0}$ if and only if it satisfies the following two conditions:

$\left(^{*}\right)$ For $E$ equipped with any of its equivalent norms, every semi-embedding of $E$ into another Banach space is an isomorphism.

$\left.{ }^{* *}\right)$ Every closed infinite-dimensional subspace of $E$ contains an infinite unconditional basic sequence.

Proof. In view of Theorem 1 and the above Remarks, only the "if" part needs a proof. So assume conditions $\left(^{*}\right)$ and $\left({ }^{* *}\right)$ are satisfied, and suppose $E$ has a closed infinite-dimensional subspace $G$ which does not contain any isomorphic copy of $c_{0}$. Applying (**), we may assume that $G$ has an unconditional basis. Since $G \not c_{0}$, this basis 
is boundedly complete ([7], Theorem 1.c.10) and hence $G$ is isomorphic to the conjugate space $Z^{*}$ of a separable Banach space $Z$ ([7], Proposition 1.b.4). We may equivalently renorm $E$ so that in the new norm, $\|\cdot\|$ say, $G$ becomes isometric to $Z^{*}$. Let $B$ be the closed unit ball of $(E, \| \cdot||)$. There is a norm $|\cdot|$ on $G$ such that $|y| \leqq\|y\|$ for $y \in G$ and $B \cap G$ is $|\cdot|$-compact. (If $\left(z_{n}\right)$ is a sequence dense in the unit ball of $Z$, then the formula $|y|=\sum_{n=1}^{\infty} 2^{-n} \mid y\left(z_{n} \mid\right.$ for $y \in G=Z^{*}$ defines a norm on $G$ which is smaller than $\|\cdot\|$ and induces the weak ${ }^{*}$-topology on $B \cap G$.) Now the formula

$$
|x|_{1}=\inf \{|y|+\|x-y\|: y \in G\}
$$

defines a norm on $E$ such that

$$
|x|_{1} \leqq\|x\| \text { for all } x \in E \text { and }|y|_{1}=|y| \text { for } y \in G .
$$

Let $B_{1}$ be the $|\cdot|_{1}$-closure of $B$. We claim that $B_{1}$ is $|\cdot|_{1}$-complete and $B \subset B_{1} \subset 3 B$.

First note that on the quotient space $E / G$ the quotient norms $\|\cdot\|^{-}$and $\mid \cdot \hat{1}_{1}$ corresponding to $\|\cdot\|$ and $|\cdot|_{1}$ are equal (and make $E / G$ a Banach space). Let $Q: E \rightarrow E / G$ be the quotient map. Now let a sequence $\left(x_{n}\right) \subset B$ be $|\cdot|_{1}$-Cauchy. Then $\left(Q x_{n}\right)$ is Cauchy in $E / G$ and hence converges to a point $\zeta \in E / G$. Since $\|\zeta\|^{\top} \leqq 1$, for each $\varepsilon>0$ there is $z \in E$ such that $\|z\| \leqq 1+\varepsilon$ and $Q z=\zeta$. Moreover, since $\left\|Q\left(x_{n}-z\right)\right\|^{\wedge} \rightarrow 0$, there is a sequence $\left(y_{n}\right) \subset G$ such that $\left\|x_{n}-z-y_{n}\right\| \rightarrow 0$; hence also

$$
\left|x_{n}-z-y_{n}\right|_{1} \rightarrow 0 \text {. }
$$

It follows that $\left(y_{n}\right)$ is $|\cdot|_{1}$-Cauchy. Since, for $n$ large enough,

$$
\left\|y_{n}\right\| \leqq\left\|x_{n}\right\|+\|z\|+\left\|x_{n}-z-y_{n}\right\| \leqq 2(1+\varepsilon),
$$

i.e., $y_{n} \in 2(1+\varepsilon)(B \cap G)$, and since $B \cap G$ is $|\cdot|_{1}$-complete, the sequence $\left(y_{n}\right)$ is $|\cdot|_{1}$ convergent to a point $y \in 2(1+\varepsilon)(B \cap G)$. Denoting $x=y+z$ we see that

$$
\left|x_{n}-x\right|_{1} \rightarrow 0
$$

and

$$
\|x\| \leqq 2(1+\varepsilon)+(1+\varepsilon)=3(1+\varepsilon) .
$$

Since $x$ does not depend on $\varepsilon$, we have $\|x\| \leqq 3$. This concludes the proof of the claim.

Finally, let $\|\cdot\|_{1}$ be the Minkowski functional of $B_{1}$. From the above proof, it is evident that $\|\cdot\|_{1}$ is a norm equivalent to $\|\cdot\|, B_{1}$ is the closed unit ball of $\left(E,\|\cdot\|_{1}\right)$, $|\cdot|_{1} \leqq\|\cdot\|_{1}, B_{1}$ is $|\cdot|_{1}$-complete and $B_{1} \cap G=B \cap G$ is $|\cdot|_{1}$-compact. Hence the identity map from $\left(E,\|\cdot\|_{1}\right)$ into the completion of $\left(E,|\cdot|_{1}\right)$ is a semi-embedding that is not an isomorphism.

Remarks. (1) Some parts of the above proof were inspired by the arguments used in the proofs of Propositions 2 and 3, and by the Remark on page 235 of [9]. 
(2) Let us recall that condition $\left(^{* *}\right)$ is certainly satisfied when $E$ is a subspace of a Banach space with an unconditional basis ([7], p. 27) or, more generally, a subspace of an order continuous Banach lattice ([8], Theorem 1.c.9).

(3) We do not know whether the condition $\left(^{*}\right)$ of Theorem 2 is equivalent to the apparently weaker one: $\left({ }^{*}\right)$ Every semi-embedding of $E$ (with the given norm) into another Banach space is an isomorphism.

\section{REFERENCES}

1. J. Diestel and J. J. UhL, Jr., Vector Measures (American Mathematical Society, Mathematical surveys, number 15,1977$)$.

2. L. Drewnowski, On minimally subspace-comparable F-spaces, J. Funct. Analysis 26 (1977), $315-332$.

3. L. Drewnowski, An extension of a theorem of Rosenthal on operators acting from $l_{\infty}(\Gamma)$, Studia Math. 57 (1976), 209-215.

4. L. Drewnowski, Un théorème sur les opérateurs de $l_{\infty}(\Gamma), C . R$. Acad. Sci. Paris, Sér. A, 281 (1975), 967-969.

5. J. HAGLer, A counterexample to several questions about Banach spaces, Studia Math. 60 (1977), 289-308.

6. N. J. Kalton, Basic sequences in $F$-spaces and their applications, Proc. Edinburgh Math. Soc. 19 (1974), 151-167.

7. J. Lindenstrauss and L. Tzafriri, Classical Banach Spaces I. Sequence Spaces (Ergebnisse der Math. 92, Springer, Berlin-Heidelberg-New York, 1977).

8. J. Lindenstrauss and L. Tzafriri, Classical Banach Spaces II. Function Spaces (Ergebnisse der Math. 97, Springer, Berlin-Heidelberg-New York, 1979).

9. H. P. Lotz, N. T. Peck and H. Porta, Semi-embeddings of Banach spaces, Proc. Edinburgh Math. Soc. 22 (1979), 233-240.

10. A. Petczyñski and Z. Semadeni, Spaces of continuous functions (III), Studia Math. 18 (1959), 211-222.

11. H. P. Rosenthal, On relatively disjoint families of measures, with some applications to Banach space theory, Studia Math. 37 (1970), 13-36.

Institute of Mathematics

A. Mickiewicz UNIVERSITY

Poznañ, Poland 